\title{
Development of crowd investing on the basis of ICO crypto assets using block-options for the supply of electric generation capacity
}

\author{
Andrew Varnavskiy \\ Financial University under the \\ Government of the RF, \\ Leningradsky Prospekt 49, \\ Moscow, Russia \\ Email: AVVarnavskiy@fa.ru \\ Vladislav Trubnikov \\ Financial University under the \\ Government of the RF \\ Leningradsky Prospekt 49, \\ Moscow, Russia \\ Email: vladtrubnikov95@gmail.com
}

\author{
Ulia Gruzina \\ Financial University under the \\ Government of the RF \\ Leningradsky Prospekt 49, \\ Moscow, Russia \\ Email: ymgruzina@fa.ru \\ Anastasia Buryakova \\ Financial University under the \\ Government of the RF \\ Leningradsky Prospekt 49, \\ Moscow, Russia \\ Email: AOBuryakova@fa.ru
}

\author{
Artur Rot \\ Wroclaw University \\ of Economics, \\ ul. Komandorska 118/120, \\ 53-345 Wroclaw, Poland \\ Email: artur.rot@ue.wroc.pl \\ Ekaterina Sebechenko \\ Financial University under the \\ Government of the RF \\ Leningradsky Prospekt 49, \\ Moscow, Russia \\ Email: EVSebechenko@fa.ru
}

\begin{abstract}
-attraction of investments into the electric power industry is complicated by a number of problems related to the long payback period and instability of the conditions on the market. Investors in the electric power industry must invest huge sums of money and hope for maintaining high demand and prices in the future in order to get a payback from the project. Decentralized investment distributes risks and allows you to raise a sufficient profit. In the paper Authors will consider the possibility of using distributed register systems to involve potential energy consumers in investing in the construction of generating facilities with a certain amount of energy at a reduced price in the future. In addition, the blockchain system allows to solve problems of the electric power market: simplify and make more flexible maintenance of transactions, automate trade settlements, reduce the risks of non-fulfillment of obligations. In turn, the improved interaction environment of market participants will make the market more stable, which will increase the investment attractiveness of the industry.
\end{abstract}

\section{INTRODUCTION}

A ccording to experts, the provision of energy generation facilities by 2023-2027 may be exhausted due to the growing demand for energy, as well as the depreciation of previously constructed facilities [1]. Existing financial instruments do not allow a broad mass of investors to participate in financing the energy sector: these are mostly utilized by professional participants. At the same time, future energy consumers are interested in acquiring it at reduced tariffs and could become anchor investors in the construction of new facilities. New instruments for attracting financing are needed, considering the high capital intensity and significant time costs for the construction of energy infrastructure. At that moment, discussions are underway to introduce the latest technologies in the energy distribution model.

Nowadays, various start-ups, such as Grid+, Power Ledger, EnergoLabs, LO3 Energy and others, are developing blockchain-based applications for the electric power industry in many countries around the world. The reason for so much attention to the technology of the distributed register in the electric power industry is the prospects of optimizing not only the process of trading in power capacities, but also the process of energy generation and distribution of power capacities.

Blockchain technology is attractive due to some of its special properties. First of all, it allows you to automate the processes of accounting and some manual operations, which reduces transaction costs. Secondly, it allows you to save previously recorded information in its original form, which increases the trust of participants to the system and helps to track the conducted transactions.

The purpose of this paper can be described as the development of the most efficient model for conducting ICO (Initial Coin Offering) of a system of blockchain-options for the supply of electric power.

Dialectic methods, methods of system analysis, general scientific empirical methods were used to study the object of scientific research and achieve its goal: comparison, analysis, synthesis, method of scientific abstraction. Methods of retrospective, current and prospective analysis and synthesis of theoretical and practical material were used to systematize the data obtained. The methods used together made it possible to ensure the reliability of the research conducted and the validity of the conclusions drawn.

The material has been prepared with the results of studies carried out at the expense of funds provided under the grant of the Bank Santander. 


\section{RELATED WORK}

The possibility of using blockchain in the electric power industry was considered by some authors, in particular, Mamontova M.U. drew attention to simplifying the interaction between generating companies and their customers through smart contracts [2]; Bogdanova E.D. and Valieva D.G. made a review of the use of distributed registry technology or the potential interest from companies in the energy sector [3]. Such big analytical companies like PwC [4] and Deloitte [5] carried out research in the field of blockchain in the energy sector and noted that the technology would help reduce the costs associated with user interaction with the audit; which will make transactions more transparent and help to establish fair prices.

According to research by GTM over the past year, blockchain start-ups attracted approximately $\$ 300$ million to the energy sector through the ICO [6]. Most projects are not currently implemented in practice, although they have identified in their studies a great potential for using the new technology. As part of the work, the documentation was reviewed Greeneum, Suncontract, Grid+, ImpactPPA, Power Ledger, EnergoLabs, LO3 Energy, Enerchain, WePower.

The introduction of modern financial technologies into the electric power industry is quite relevant and popular today on the market. For the purposes of this study, we analysed the works of a number of foreign authors. Thus, our team utilized the article "Security and Privacy in Decentralized Energy Trading through Multi-Signatures, Blockchain and Anonymous Messaging Streams", Nurzhan Zhumabekuly Aitzhan, Davor Svetinovic in which the authors discuss the problem of securing transactions in decentralized energy trading. They also proposed a concept for a decentralized energy trading system using blockchain technology. [7] Some provisions of this concept are reflected in the model developed by us.

You can also find traces of the following scientific works in the paper "Blockchain technology in the chemical industry: Machine-to-machine electricity market" by J. Sikorski, J. Haughton, M. Kraft (an example where blockchain is employed to establish a M2M electricity market in the context of the chemical industry) [8]; "A blockchain-based smart grid: towards sustainable local energy markets" by E. Mengelkamp, B. Notheisen, C. Beer, D. Dauer, C. Weinhardt (an example of decentralized market platform for trading local energy generation) [9]; "Industrial Blockchain Platforms: An Exercise in Use Case Development in the Energy Industry" by J. Mattila, T. Seppälä, C. Naucler, R. Stahl et al. (a case for autonomous machine-to-machine transactions of electricity in a housing society environment) [10].

During the process of finding technological solutions our team has used an experience of well known experts and communities, who developed smart contracts [11]. An external source of code and technological pattern was taken in order to construct and plan the synchronization system [12].

It was suggested that a decentralized platform could be built based on the analysis of the Russian electricity market, integrating the advantages of options and ICO, to form a model in which both future consumers and private investors could participate in financing the construction of new facilities and granting access to cheaper energy.

\section{RESEARCH OF ACTIVE PROJECTS, WHICH UTILIZE BLOCKCHAIN IN ENERGY SECTOR}

The study of existing energy blockchain projects allowed us to identify the two most successful investment strategies Grid+ and WePower solutions. The key factors were: signed agreements with energy companies, the availability of tested technological solutions and active marketing policy. However, it is still difficult to talk about the effectiveness of the projects reviewed in terms of comparing costs and financial results, since they are not fully implemented at the moment. Nevertheless, theoretically the benefits to suppliers and consumers are undeniable. It is established that the liquidation of intermediaries and P2P calculations will inevitably lead to a reduction in energy prices in a highly competitive environment.

One of the main advantages of the blockchain model is that all energy can be accurately recorded for certain suppliers and consumers. The control of the distributed and consumed energy makes it possible to ensure its optimal use. At the same time, a simplified distribution process, where consumers directly interact with producers, facilitates the free formation of prices in the market. On the one hand, a decrease in transaction costs will lead to a drop in prices, but on the other hand, the opposite effect is possible. For example, in the case of significant differentiation in producer costs, the price will not necessarily tend to average or minimize. As a result of the insufficient maturity of the digital market and the possibility of consumers sticking to the old redistribution system, the only loyal consumers will remain on decentralized sites. So, depending on the structure and volume of the digital energy market, the prices of the traded commodity will fluctuate.

The usefulness of derivatives in energy supply depends not only on the general provisions of the pricing mechanism, but also on the specifics of the market. Based on the fact that the option is intended to solve a well-defined set of tasks, you must be fully aware of the energy platform. Technological features in some cases can provide price stability and minimize the risks that lead to a lack of a need for options. Assigning certain rights and obligations to the token will inevitably lead to a restriction of its functionality. By itself, the integration of ICO and options is not the goal, it is only one of the possible tools for optimizing the interaction of market participants. Therefore, it is impossible to consider the methods of financing a project without an accurate idea of the specifics of the decentralized system.

Today tokens produced during the ICO can perform a variety of functions - payment for goods, works, services provided by the company; various discounts and bonuses; means of payment; system access keys; confirmation of ownership of an asset or a normal means of attracting financing and etc. For example, a token is the accounting 
unit of a system whose properties depend solely on how the community will use it. Tokens can be secured with property and have a constant inflation rate. The company can also provide some monetary policy with it. The number of tokens produced by projects is also not limited, which allows creating different models that meet the requirements of the platform.

The following conclusions were made while analyzing existing energy projects on the market, regarding the efficiency of their use:

\section{A. Projects with one token: WePower, Suncontract, Greeneum}

It was possible to establish that using tokenization in these projects does not exclude speculation on the exchange rates of the coins on the platforms. The offer of the token will depend wholly and entirely on the number of users providing and purchasing energy. The more suppliers will be there, the more coins and the lower the price in conditions of weak demand. However, in the case of a very limited supply and high demand, attempts to dishonest fraud in the market cannot be ruled out, when profits will be used by suppliers for speculative purposes. In this case, the transformation of the token into a contract (option) would make sense. Nevertheless, it cannot be asserted that the use of derivative financial instruments will not exacerbate the situation in the long term and will not lead to even greater volatility.

\section{B. Projects with two tokens: Grid+, Power Ledger, EnergoLabs, ImpactPPA, Exergy.energy}

The model with two tokens was recognized as the most effective, based on the analysis of the decentralized companies providing energy distribution and trading services, which allows to distinguish between trading on the exchange (coin / national currency) and intra-system trade (coin / energy). This approach excludes the possibility of speculation, because each platform user can exchange a token only for energy. At the same time, minimization of risks of the parties in models with two tokens occurs due to the formation of a reserve fund, which can be used in the event of an emergency: a sharp increase in the costs of production of a supplier or a sudden drop in the solvency of consumers. The difference between the price of supply and demand will be offset by the resources accumulated by the system.

\section{Models which do not imply tokenization of energy: Enerchain}

The site allows to trade in gas, solar, wind and other energy without intermediaries. The expediency of the option in this case will depend on a number of factors, but the main one is the technological features of the platform. The combination of a market mechanism for pricing and the limited choice of consumers when pooling energy suppliers can lead to undesirable consequences. In fact, there will be a situation when agents of the offer will be able to "plan ahead" the profit received from the exercise of the option.
The solution of this problem can lie in the plane of improving the technological features of the platform.

\section{IMPLEMENTATION OF AGENTS`INTERRELATIONS MECHANISMS DURING THE WORK OF THE PLATFORM}

The results of the analysis of the effectiveness of various option models, as well as the formed view of the appropriateness of holding options for the supply of power capacities, made it possible to draw up a model for the interaction of the parties. The proposed solution combines the advantages of previously analyzed projects and is designed to minimize the likelihood of instability in the energy market. Given the importance of the investment component, it was decided to form a blockchain model with three types of tokens:

1. An external token (ET), which is a unit of account. It is traded on a crypto exchange and can be exchanged for an internal or investment token of a vendor.

2. Internal energy tokens of individual suppliers $(\mathrm{T} 1, \mathrm{~T} 2, \ldots$, Tn), which is necessary for energy trading, call and put options. Each internal token is provided with a certain amount of generated energy, which allows investors to guarantee the right to receive it in the future. At the same time, the token which identifies the energy will serve as a marker confirming the fact of the transaction.

3. The investment token (I1, I2, ... In) - accounting unit for attraction of financing.

The conclusion has been drawn based on the results of the analysis. It is necessary to develop a qualitatively new model of energy tokenization. It is needed to preserve the advantages of using derivative tools when integrating with blockchain systems. The expediency of options in the model is mainly determined by the possibility of coordinating the interaction of market participants, rather than purely financial provisions. The relationship of rights and obligations, guarantees and market freedoms play a decisive role.

During the development of the decentralized platform model, special attention was paid to the risk-oriented approach. The risk assessment made it possible to identify the following possible sources of risk: legal regulation, investment attractiveness of the project, participation of third parties, hackers and the human factor. At the same time, risk factors were justified: the volatility of the crypto-currency, the electricity costs for the PoW protocol, the predominance of the role of large investors, the protection of personal data.

\section{A. Market clearing mechanism}

The general scheme of the proposed platform consists of several key elements. At the initial stage, the supplier receives a certain number of internal tokens $\mathrm{T} 1, \mathrm{~T} 2, \ldots, \mathrm{Tn}$, which is equal to the amount of energy generated by him, which he is ready to send to the system. These tokens are placed on the internal trading platform of the system, paired with an external token. That is, in order to acquire energy, the consumer must first purchase an external platform token on the common exchange, and then exchange it for the energy / internal token of the particular company (Fig. 1). 


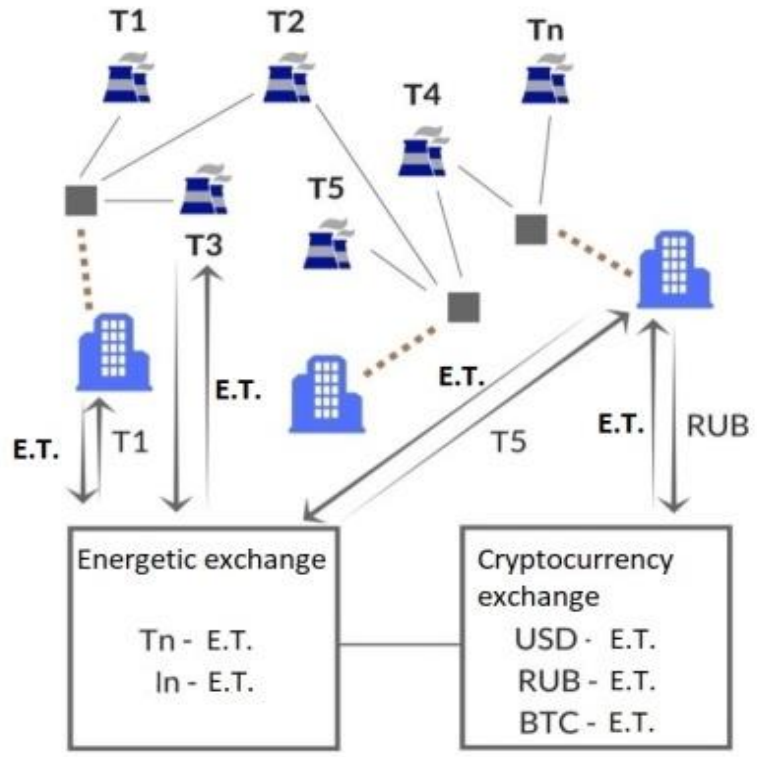

Fig. 1 Diagram of movement of external and internal tokens on the energy platform

In this case, clearing mechanism is based only on decentralized P2P interaction. Buyers can choose on the domestic exchange any token with a smart contract included in it. This coin has fixed the price and date and volume of delivery, established by the seller. The moment of buying the energy token on the domestic exchange will signify the beginning of the transaction on the blockchain option between the buyer and the energy supplier.

In addition, there is possible a following situation: when the energy buyer sets the terms of the smart contract and publishes it on the internal exchange, it can be prescibed to any generating company, which bought it.

The concept of the Waves project was taken as the basis of the internal stock exchange platform. The Waves developers created it as a decentralized exchange of tokens, sometimes abbreviated as DEX (Decentralized Exchange), due to the advantages of this type of system. DEX does not create limits for any transactions. After the process of buying cryptocurrency, it appears in your wallet instantly. Also, the exchange charges a very small fixed commission for each order. Transaction security does not cause fear among users, because the funds are stored in your wallet, not in the exchange. There is also no risk of losing money, as it may happen in a centralized exchange, but users still receive high bandwidth transaction channels and a centralized exchange node in order to maximize transaction speed.

Decentralized cryptocurrencies are traded on all centralized exchanges. Bitcoin and other cryptocurrencies are the ideal goods to steal. Transactions are not reversible; transfers are difficult to trace. Centralized exchange of cryptocurrencies is not sustainable in the long term, since the cost of a successful attack can spoil any successful exchange.

On the other hand, while there is a very high transaction capacity, the site will not be able to have a convenient solution for a fully decentralized trade. The inclusion of all trading data in the blockchain is feasible, but then you have to deal with the delay of the lock and the set of data that must be synchronized across all nodes.

The solution can be simple - a decentralized block system, with a centralized order of checking the blocks. That is, there is a server that corresponds to incoming orders, but does not have access to resources. There is no chance that the user will be able to lose money in this setup, since he has full control over the funds. When the server finds the corresponding pair, it initiates the transfer to the block that moves the funds. Correctness of compliance is verified in a decentralized manner, and no funds can be transferred if orders are not verified (Fig. 2).

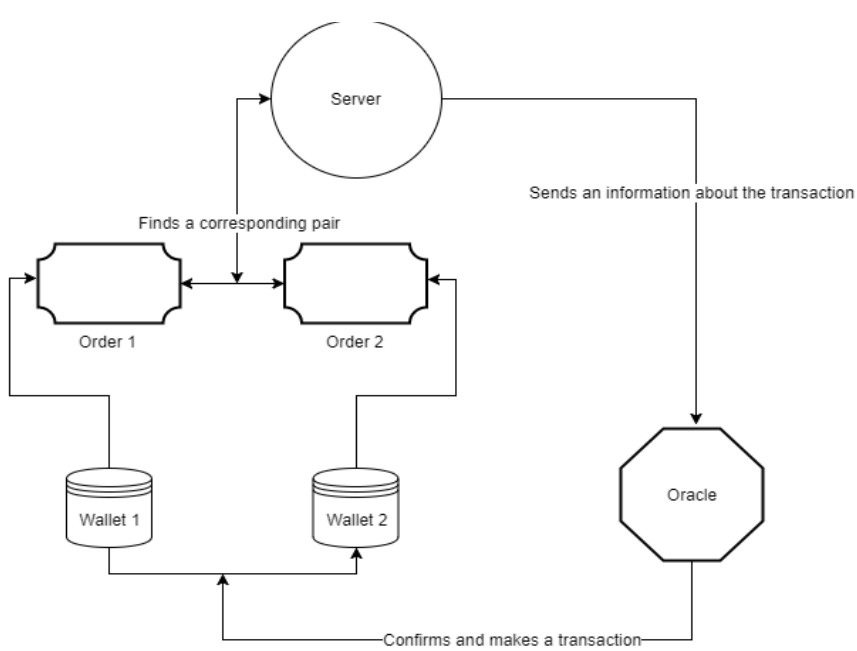

Fig. 2 Scheme of transactions inside the exchange

In accordance with the developed model, each of the energy companies or the directly generating stations, gets the opportunity to issue their own tokens in order to attract financing for the construction of new facilities or the updating of the existing ones. The exchange of each of the tokens I1, I2,..., In also occurs on the internal power exchange platform using the mechanism, similar to the process of buying an internal token. Consumers, in turn, get tokens by investing in the project. Those tokens are supported by energy, which they can exchange in the future for internal tokens T1, T2,..., Tn with discount by making a real option contract with the supplier, or exchange tokens for external coins (Fig. 3).

Often, energy companies, especially from the small and medium-sized business segment, find it difficult to attract investment to build or improve the electricity distribution infrastructure. Therefore, the platform envisages the creation of separate contracts to attract funds at the request of specific organizations. Thus, local residents in small settlements will have the opportunity to pay for the construction of power plants and power lines at their own expense or by third-party investors. The implementation of transactions can be made through the trade balance, with already available tokens of the particular company, bought at the rate for the E.T. 


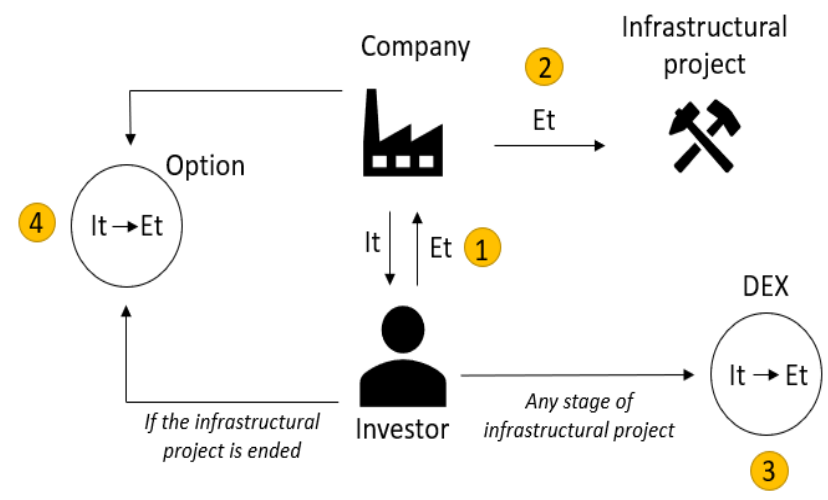

Fig. 3 The system of attracting investment - a general view

In the developed model options based on smart contracts allow you to build a clear scheme of interaction between the parties, delineating the rights and obligations. With the smooth operation of the system and full automation, it becomes possible to use options as tools to ensure the predictability of the energy market. All the transactions made at any time can be recorded on the platform, which means that it will be possible to track daily, seasonal and other fluctuations, providing information to suppliers for the future planning of power generation. The developed model of the decentralized platform seems to be the most optimal taking into account the requirements of the market and the level of development of modern technologies.

From a technological point of view, the proposed model for interaction between counterparties of a decentralized energy area includes the following participants:

1. The token creator, who creates a special token to ensure that the above-described smart contracts and the system as a whole are working optimally. Generating two contracts requires significant resources in Gas units on the Ethereum platform. As a result, the creator will be forced to absorb part of the costs when using smart contracts.

2. The Seller of the option is the user who enters the ERC20 tokens in the amount indicated in the contract price. The option maker pays a commission.

3. Option buyer - the user who buys an option contract in exchange for the price set by the seller in the ERC-20 token through a protocol on the platform.

It is assumed that the option contract will give the right to receive special (ERC-20) tokens in exchange for the external ERC-20 tokens during the period before the contract expiration date at the strike price. A transaction using this right is called an "executive" transaction. A certain trusted third party that provides data on the current market condition in real time can be replaced by a platform administrator or by mutual agreement of its participants.

Blockchain allows to simplify and make more flexible the clearing system in the market of electricity sales. The decentralized exchange is the place of "meeting" of sellers and buyers, where everyone can interact with each other to find the best deal possible. The choice of price will help to make the transaction ledger on the blockchain, which will show the price trends.

\section{B. Block-options' payment system}

Two types of tokens will be used in oder to conduct options - internal (T1, T2, ..., Tn) and external (Fig. 4). Call option, where the basic asset is electricity, is formed by the supplier. The consumer receives the right to supply energy at the specified time at a certain strike price after payment of the premium for the option. If the market price exceeds the exercise price at the moment of execution, the consumer realizes his right by paying the supplier a stipulated amount in external tokens. External tokens received by the supplier can be immediately sold on the exchange for other currency.

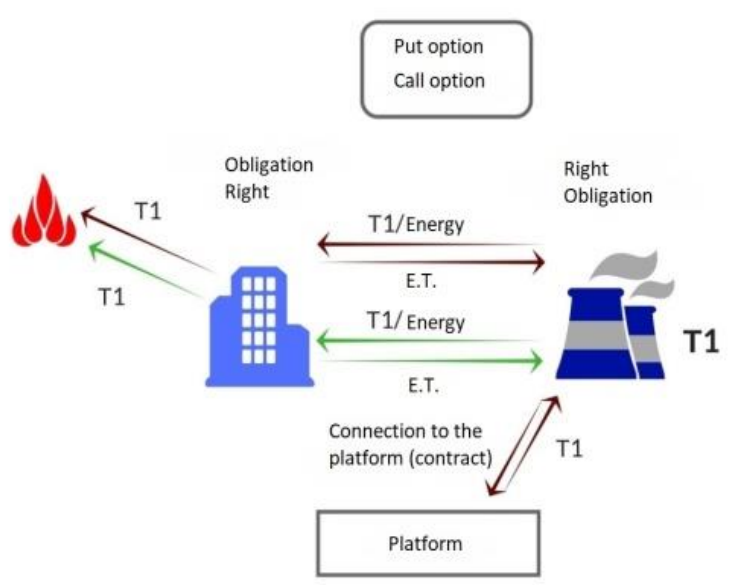

Fig. 4 Scheme of holding block of options for the supply of power capacity

The order of put option will be mirrored from the call option. In view of the need to formulate precise conditions for smart contracts, the flow of energy must also be accompanied by tokens. By connecting to the platform and providing information about the capacity of the power plant, the supplier receives a certain number of energy tokens $\mathrm{T} 1$, $\mathrm{T} 2, \ldots \mathrm{Tn}$, which can also be used to participate in the put option. Each energy consumer, in turn, is entitled to form put option, indicating the desired date of energy supply and the amount for which he undertakes to purchase it. In order to confirm participation in the put option the provider transfers to the consumer account a premium in form of external tokens. When the option date is reached, the supplier decides whether or not to exercise his right. Accordingly, if the execution price is higher than the market price, the supplier confirms the transaction by transferring energy tokens identifying the energy to the consumer's account. Once this condition is met, the smart contract writes off the exercise price from the consumer's account. The internal energy token after the transaction is burned, confirming the fact of sending energy.

At the same time, the platform will be able to implement the bull call spread option, which reflects the strategy when an agent buys call options for a certain price and at the same time sells a call option for the same product, but at a higher price. It is used when an agent expects an increase in the price of goods. Additional add-ons to the existing system and a special user interface are needed to support the operation of such a mechanism on the basis of smart contracts. In addition, such a contract will need to control 
the lower and higher price limits in order to activate itself if the latter is reached. To do this, it periodically updates the data from the market base of commodity prices (Fig. 5).

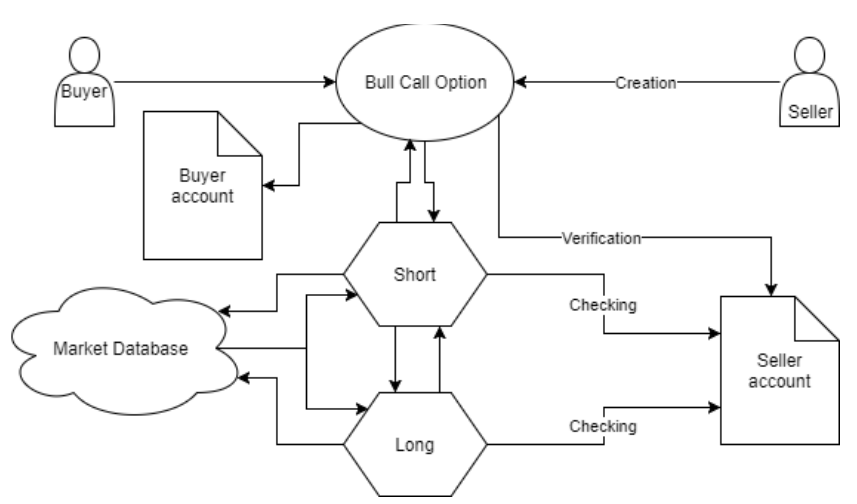

Fig. 5 Call spread option - detailed scheme

To determine this problem, it is necessary to raise the question: when and where is the code for the smart contract works? There are two different circumstances. Each smart contract is first performed by one or several miners, nodes that repeatedly offer new blocks to the blockchain. When the miner creates a block, he selects a sequence of user requests and performs associated contract codes for each Ethereum transaction in sequence, converting the old contract state to a new one. Then, it records both the sequence of transactions and the new state in the block, and suggests it for inclusion in the blockchain.

Later, when the block is added, each smart contract is reexecuted by validators: nodes that restore (and check) the current state of the block. When the validator acquires each subsequent block, it repeats each transaction to verify that the initial and final states of the block match each other. Each miner checks the blocks proposed by other participants, and the senior unit is checked by newly connected miners or clients requesting the status of the contract. Code execution for validation significantly exceeds the execution of the code for mining [13].

The speed and coherence of the platform is very important for the acquisition of popularity among the customers of the market. Each smart contract must periodically update its state and check itself with the database. Unfortunately, the Ethereum Foundation, on which the developed platform is based does not have the tools to keep the smart contract network constantly updated, and attempts to add scheduled events to each element will only increase the server load and weight of the user interface. On the other hand, there is an Ethereum Alarm Clock system that is capable of this, but its work is based on the community of the network, and therefore it is not suitable for solving the tasks of the proposed platform, since it does not provide accurate data for the activation of smart contracts.

To solve this problem, we need a separate mechanism for updating contracts (Fig. 6). Creating a special smart contract that will connect all the other operating system elements to the main server is one of the solutions. Of course, he will consume Gas, which will have to be compensated by commissions for the platform participants. Such server will help to regularly update price information and assist smart option contracts throughout their work.

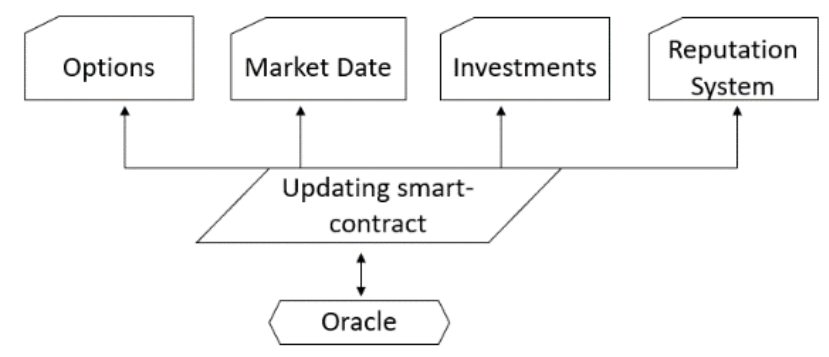

Fig. 6 Description of the work of the smart contract for updating the platform

However, Ethereum is already working on a full-fledged system for registering updates and accounting of time in smart contracts, which will avoid the listed difficulties associated with the development of its own solution.

To maintain the workability of the platform, it needs a source of finance. The main contender for this function is the system of commissions to work for the creation and execution of smart contracts at the Ethereum Foundation. Each smart contract requires a certain payment in the equivalent of Gas. This is the internal crypto-currency of Ethereum, through which the cost of production and placement of the contract is on the platform is paid. Therefore, when placing such a tool in our system, a certain amount of money will be written off in the form of tokens.

Many projects to date show that programming and the use of financial instruments on Ethereum is quite possible. However, while the underdevelopment of the structure makes the creation of such projects, cumbersome and complex, those solutions can be used in the market and give companies the necessary and unique functionality that makes them more successful than their competitors.

For the proper work of financial instruments, it is necessary to establish a communication with external sources of data on the market and counterparties. Unfortunately, at this moment, there are no reliable suppliers of such information such as Bloomberg on blockchain technology.

However, there is a project called Ethereum Price Feed, under the leadership of Roman Mandeleile, which can be used as a third-party source for smart contracts. On the other hand, this platform is updated. To solve this problem, several options were considered, including the one described above, but in the end of the simplest answers was chosen to use data from open sources such as coinmarket.com and other websites analyzing quotes of crypto-currencies online. Also, to get the prices of commodities, you can use Bloomberg or other local sources, depending on the location and methods of using the platform. These data will be used 
as the main indicators for smart contracts and, accordingly, users, too.

Sources of price data can work much better in closed blockchain systems. As with any other similar platforms, the market and industry indicators can be defined and tailored to industry standards. Providers will not be able to. Platform participants can also enter their data on the state of markets and if they are confirmed by other agents, their use becomes possible within the framework of a particular project.

\section{Interaction risks' decrease}

To implement a full-fledged trading platform requires a base in the form of a website or an application for a user interaction between each other and the blockchain system as a whole. In addition, one cannot force people to use their wallets, as this will involve third parties. That's why every user of this platform will need to open his / her own trade balance directly on the platform. The numbers of these trade balances will be used by the system as input for settlement through smart contracts.

Once one of the users decides to open such an account, he will be asked to put on a certain amount of money. This is designed to protect participants 'wallets, since a smart contract is the right to manipulate users' funds on their trade balances. Subsequently, such a contract can withdraw tokens directly from accounts of counterparties. In the future, to ensure the protection against intruders, smart contracts can be connected to the system for determining and recording the reputation of participants. Contracts also provide access only to persons who have made a transaction on their basis, which makes the intervention of the third party unlikely.

A special section will be opened on the website and in the project in order to promote and regulate the market. It will allow users to communicate with each other and influence the fate of the project as a whole and the decisions of local companies in particular.

However, this approach seems ineffective, since most users can ignore the platform's capabilities, leaving room for intruders. The special reputation system will follow to control the regulation, which is also tied to contracts and trade balances of market participants.

Another practical solution will be the creation of a reputation accounting system [14]. Systems of accounting for the reputation of users are not new on the blockchain market. There are many similar projects based on Ethereum, such as Augur and Gnosis. They are based on the value of money and hold important information. Participants in these projects receive more tokens if they have a good reputation among other users.

The main part of this system will be reputation points. In essence, they are similar to the credit score. When a person takes out a loan for the first time, he has no history, so at the beginning each agent will be given a certain "starter" rating, which will be slightly above zero.

Each creator or buyer of options has an open profile in the community forum, the data from which are displayed in the authorship of the contract. Thus, any participant will be able to put this or that rating to another user and this data will be stored in the blockchain network.

Of course, one should not forget about the possible abuse of the system, but this problem has already been partially resolved, since each new agent of the platform is obliged to make a minimum deposit for making transactions on the trading floor, which makes the creation of additional balances at least unprofitable.

Also, the system will take into account each user in order to make buyers, sellers and investors more significant if they have a good reputation among the community. This function will prevent Sybil type attacks on the blockchain network.

Another side of the reputation system will be the valuation of the energetic companies. The risk rating will be applied to them, and the profitability of the company. Also there will be a data on payments on investment shares and the total profitability ratio.

For example, the reputation area was taken from 0 to 1000 rating units, where 0 is the worst and 1000 is the best. Weight in this model was not specified in order to simplify the presentation.

Depending on the behavior of each individual agent, the community will be able to identify both conscientious and unfair users.

Such a system will allow rewarding the participants with the best reputation, giving them the opportunity for further development and opening up new market sectors. Over time, this will create an environment in which such platform users can, even if it is a local supplier or consumer.

The system also plans to take into account the total number of transactions and contracts. Continuing to use the platform, the reputation of the actors will be constantly updated, giving a chance to agents with a bad reputation to correct themselves and showing the trend and the history of everyone.

\section{V.CONCLUSION}

Thus, the following main results of the study were obtained:

- Evaluation of the effectiveness of blockchain technologies in the electric power supply industry;

- The technological and market justification for the release of several interconnected tokens on the platform;

- Proposals were made to establish relationships between contractors based on the platform;

- The method of implementation on the platform of specialized smart contracts.

The practical significance of the research work is to compare methods of applying traditional derivative financial instruments and blockchain options in the energy supply industry with the subsequent creation of the best model for the development in this sector.

Summarizing the above, based on the results of the work, the most effective model for Initial Coin Offering was developed for the system of blockchain options for the supply of electric power. Also, the principles for the functioning of 
the decentralized site were formulated during the research Realization of the proposals will allow, on the one hand, to optimize the process of trade in power capacities, and on the other hand, will lead to an increase in the efficiency of the generation and distribution of electric power.

\section{APPENDIX}

A list of terminology that was used in the paper:

Distributed ledger - A distributed ledger (shared ledger, or distributed ledger technology, DLT) is a consensus of replicated, shared, and synchronized digital data geographically spread across multiple sites, countries, or institutions. There is no central administrator or centralized data storage.

Blockchain - A blockchain, originally block chain, is a continuously growing list of records, called blocks, which are linked and secured using cryptography. Each block typically contains a cryptographic hash of the previous block, a timestamp and transaction data.

Smart contract - A smart contract is a computer protocol intended to digitally facilitate, verify, or enforce the negotiation or performance of a contract. Smart contracts allow the performance of credible transactions without third parties. These transactions are trackable and irreversible.

ICO (Initial Coin Offering) - An unregulated means by which funds are raised for a new cryptocurrency venture. ICO is used by startups to bypass the rigorous and regulated capital-raising process required by venture capitalists or banks. In an ICO campaign, a percentage of the cryptocurrency is sold to early backers of the project in exchange for legal tender or other cryptocurrencies (usually for Bitcoin).

Call option - A call option, often simply labeled a "call", is a financial contract between two parties, the buyer and the seller of this type of option. The buyer of the call option has the right, but not the obligation, to buy an agreed quantity of a particular commodity or financial instrument (the underlying) from the seller of the option at a certain time for a certain price (the strike price). The seller ("writer") is obligated to sell the commodity or financial instrument to the buyer if the buyer so decides. The buyer pays a fee (called a premium) for this right. The term "call" comes from the fact that the owner has the right to "call the stock away" from the seller.

Put option - In finance, a put or put option is a stock market device which gives the owner of a put the right, but not the obligation, to sell an asset (the underlying), at a specified price (the strike), by a predetermined date (the expiryor maturity) to a given party (the seller of the put). The purchase of a put option is interpreted as a negative sentiment about the future value of the underlying stock. The term "put" comes from the fact that the owner has the right to "put up for sale" the stock or index.

\section{REFERENCES}

[1] F.V. Veselov, A. Khokhlov, Internet of Energy: how distributed energy will affect security, prices for electricity and ecological situation, Russian version of Forbes, section "Business", October 18, 2017 [In Russian]

[2] M.J. Mamontova, "Blockchain and opportunities of its implementation in energy", Information technology in science, management, social sphere and medicine, Collected scientific proceedings of IV International scientific conference, December 5-8 2017, Tomsk - pp. 417-419 [In Russian]

[3] E.D. Bogdanova, L.G. Valieva, "Cryptocurrency and energy", Problems, prospects and tendencies of innovative science development: collected articles of international academic and research conference, 2017, pp. 63-67 [In Russian]

[4] PwC, Use cases for blockchain technology in Energy \& Commodity trading - 2017, [online] Available at: https://www.pwc.com/gx/en/ industries/assets/blockchain-technology-in-energy.pdf [Accessed 2018]

[5] Deloitte, Blockchain applications in energy trading, 2016, [online] Available at: https://www2.deloitte.com/global/en/pages/energy-andresources/articles/role-of-blockchain-in-the-energy-and-resourcesindustry.html [Accessed 2018]

[6] CCN, Energy Sector Invests $\$ 300$ Million In Blockchain In Past Year, [online] Available at: https://www.ccn.com/energy-sector-invests300-million-in-blockchain-in-past-year/ [Accessed 2018]

[7] Aitzhan, N. Zhumabekuly, and D. Svetinovic, "Security and privacy in decentralized energy trading through multi-signatures, blockchain and anonymous messaging streams", IEEE Transactions on Dependable and Secure Computing, 2016

[8] J. Sikorski, J. Haughton, and M. Kraft., "Blockchain technology in the chemical industry: Machine-to-machine electricity market”, Applied Energy No. 195 (2017), pp. 234-246

[9] E. Mengelkamp, et al., "A blockchain-based smart grid: towards sustainable local energy markets”, Computer Science-Research and Development no. 33.1-2 (2018): 207-214

[10] J. Mattila, et al. "Industrial blockchain platforms: An exercise in use case", Development in the energy industry no. 43. The Research Institute of the Finnish Economy, 2016

[11] Anon, (n.d.). Smart Contract Application Examples and Use Cases .... [online] Available at: https://www.draglet.com/blockchainapplications/smart-contracts/use-cases [Accessed 2018].

[12] Anon, (n.d.). Smart Contract - A Fully Decentralized Oracle Network. [online] Available at: https://link.smartcontract.com [Accessed 2018].

[13] Arstechnica. Bitcoin's insane energy consumption, explained [online]: URL:https://arstechnica.com/tech-policy/2017/12/bitcoinsinsane-energy-consumption-explained/

[14] M. Kindy, Divine: A Blockchain Reputation System For Determining Good Market Actors https://medium.com/topl-blog/divine-ablockchain-reputation-system-for-determining-good-market-actors7c47a0308ae/ [Accessed 2018]. 